\title{
Where is the Interface of the Stratocumulus-Topped PBL?
}

\author{
C.-H. Moeng \\ National Center for Atmospheric Research,* Boulder, Colorado \\ B. STEVENS \\ Department of Atmospheric Sciences, University of California, Los Angeles, Los Angeles, California \\ P. P. Sullivan \\ National Center for Atmospheric Research, * Boulder, Colorado
}

(Manuscript received 10 August 2004, in final form 19 October 2004)

\begin{abstract}
Various locally defined (not horizontal mean) interfaces between the stratocumulus-topped PBL and the free atmosphere are investigated using a fine-resolution large-eddy simulation with a vertical grid spacing of about $4 \mathrm{~m}$. The local cloud-top height is found to be always below the height where the maximum gradient of the local sounding occurs, and the maximum-gradient height is always below the interface where PBL air can reach via turbulent motions. The distances between these local interfaces are of significant amount, a few tens of meters on average. Air between the cloud-top and maximum-gradient interfaces is fully turbulent, unsaturated, but rather moist. Air between the maximum-gradient and turbulent-mixing interfaces consists of turbulent motions that are intermittent in space and time. The simulated flow shows no clearly defined interface that separates cloudy, turbulent air mass from clear, nonturbulent air above, even locally.
\end{abstract}

\section{Introduction}

The cloud-top interface of a stratocumulus-topped PBL (STBL) moves up and down in space and time in response to underlying turbulent motions; the amplitude of this undulation is typically several tens of meters, depending on the strength of the underlying turbulence and the overlying temperature inversion. Because cloud top is a visible interface, it is often used in aircraft research deployments to locate flight levels. For example, cloud-top penetration flight legs, which fly at a nearly constant height in and out of the cloudtop undulation, are used to study the properties of and jump condition across the cloud-top interface (Len-

\footnotetext{
* The National Center for Atmospheric Research is sponsored by the National Science Foundation.
}

Corresponding author address: Dr. Chin-Hoh Moeng, MMM Division, NCAR, P.O. Box 3000, Boulder, CO 80307-3000.

E-mail: moeng@ucar.edu schow et al. 2000). Cloud-top interface is also used as a reference frame in mixed layer theory. For example, Randall (1980) assumes that the turbulent, saturated air mass is "separated from an overlying, quiet, unsaturated layer by a thin transition layer, across which the temperature, mixing ratio and turbulence intensity may change sharply." He uses this framework to derive a cloud-top entrainment instability (CTEI) criterion in which mixtures of these two distinct air masses become negatively buoyant in their surrounding unmixed cloudy air. In recent papers, Lilly (2002a,b) also argues that "the interface (between the mixed layer and the upper environment) is usually quite abrupt locally" and by averaging over a coordinate system following the local cloud-top height, the statistics of averaged quantities (such as mean liquid potential temperature, $\Theta_{l}$, and water mixing ratio, $Q_{t}$ ) should preserve their sharp jump across the interface similar to local soundings. Lilly uses this framework to derive an entrainment-rate formula and a new CTEI criterion. Implicit in Randall's and Lilly's arguments (and perhaps in many STBL 
studies) is that the cloud-top interface is the height where the sharpest jump in local soundings occurs, where turbulent motion is separated from the freeatmospheric nonturbulent flow, and where entrainment takes place.

In this study we ask, how valid is this idealization and are the cloud-top interface, maximum-gradient (sharpedged) interface and turbulence interface the same? If not, how do they relate to each other? These interfaces are defined as follows: the cloud-top interface $z_{\mathrm{lwc}}$ is the surface that separates saturated and unsaturated air; the maximum-gradient interface $z_{\mathrm{mgd}}$ marks the largest (vertical) gradient of $\theta_{l}$ or $q_{t}$ local soundings; and the turbulent-mixing interface $z_{\text {mix }}$ is defined as the upper boundary where PBL air can reach via turbulent motion. (Among these interfaces, $z_{\text {mix }}$ is perhaps most representative of the entrainment interface since by definition entrainment is a process where laminar flow is brought into and subsequently becomes part of the turbulent flow.) The heights of all three interfaces vary in space $(x, y)$ and time $(t)$. We use the large-eddy simulation (LES) approach, which explicitly calculates the time-dependent and three-dimensional energy-containing turbulent flow, to investigate the relationships among these interfaces.

\section{Method}

Our LES flow field was based on the first flight (RF01) measurements of the Second Field Experiment of Dynamics and Chemistry of Marine Stratocumulus (DYCOMS-II). The stratocumulus cloud during RF01 was solid and persistent, as reported by Stevens et al. (2003); it was measured at night and hence included no solar radiation effects. Our LES setup was similar to the seventh intercomparison study performed by the GEWEX Cloud System Study (GCSS) BoundaryLayer Cloud Group, which was detailed in Stevens et al. (2005).

The grid points we used in this study were $200 \times 200$ $\times 400$ covering a numerical domain of $3.36 \mathrm{~km} \times 3.36$ $\mathrm{km} \times 1.5 \mathrm{~km}$; thus the grid size was about $17 \mathrm{~m}$ in the horizontal and about $4 \mathrm{~m}$ in the vertical. Although our current standard LES code uses the pseudospectral method for all horizontal advection terms and a fluxlimited upwind algorithm (a monotone scheme) for vertical advection of scalars (Sullivan et al. 1998), in this study we also applied a flux-limited algorithm to horizontal advection of scalars. Moeng et al. (2004) showed that using the pseudospectral scheme for horizontal advection of scalars resulted in spurious overshoots of temperature and moisture at the interface, since sharp gradients exist in all three directions of a resolved undulating interface. These overshoots would have produced undesired small-scale features at the interface. Using a monotone scheme for the horizontal advection of scalars avoids this spurious feature, at the price of more localized numerical diffusion. However, we also analyzed LESs with different numerics and subgridscale models and found the major features we discuss in the next sections to be insensitive to the treatment of numerics and small-scale motions.

The turbulence in the DYCOMS-II RF01 STBL was driven by cloud-top radiative cooling, surface heating, latent heating, and some wind shear. As in Stevens et al. (2005), infrared radiation (IR) flux was computed using a three-term formulation, where the first term represents the cloud-top cooling just below the cloudtop interface, which is believed to be the major driving force for STBL turbulence; the second term for cloudbase warming; and the last term for IR cooling above the cloud-top interface. We examine the sensitivity of our results to the second and third terms.

With the LES flow field, it is straightforward to calculate the cloud-top interface and the maximumgradient interface. Cloud-top interface $z_{\mathrm{lwc}}$ was derived as the uppermost grid point (along each grid column) where the liquid-water mixing ratio changed from nonzero to zero. The maximum-gradient interface was calculated by searching for the height of maximum vertical gradient of $\theta_{l}$ again along each vertical column of every instantaneous LES volume. We found that the locations of the maximum $\theta_{l}$ gradient and the maximum $q_{t}$ gradient were about the same.

Locating the turbulent-mixing interface was not as straightforward. We added two passive tracers to the LES flow (after the turbulence was well established) and used them to define the top of the turbulent layer as follows: scalar $A$ was released in the lower half of the mixed layer; as a result of turbulent mixing it filled the whole turbulent layer after a few large-eddy turnover times. Scalar $B$ was released slightly above the STBL, and as the STBL grew, it entrained into the turbulent layer. To keep scalar $B$ above the turbulent layer, we included a simple decay term $-A B / \tau$ in the conservation equation of $B$ so that soon after scalar $B$ was entrained and mixed in with scalar $A$, it decays. For $B$ to decay quickly upon contact with $A$, we set the decay time scale $\tau$ to a very small value $(0.1 \mathrm{~s})$, much smaller than the large-eddy turnover time of about $10 \mathrm{~min}$ in this case. (The initial concentration of both scalars was arbitrarily set to 1 at their source regions and 0 elsewhere. Because of the use of a nonbackward scheme, the decay term can lead to negative concentrations of 
$B$, and hence a minimum concentration of zero was imposed. Admittedly, this clipping method was arbitrary, but the tracer calculation was performed only for a few large-eddy turnover times and the results were used solely for the purpose of diagnosing $z_{\text {mix }}$.) The surface $z_{\text {mix }}$ was calculated as the upper boundary of scalar A (where the concentration changes from nonzero to a negligibly small value 0.0001 ), or the lower boundary of scalar $B$. The two interfaces computed from scalars $A$ and $B$ are almost the same, as shown below.

We first ran the LES for $\sim 90$ min to establish a fully developed turbulent flow. We then released scalars $A$ and $B$ into the LES flow field and continued the LES for another $30 \mathrm{~min}$ (2-3 large-eddy turnover times). The following analysis was taken from the LES at the end of the 2-h simulation; the area coverage by cloud was about $93 \%$ and the mean mixed layer top (defined as the horizontal mean of $z_{\mathrm{mgd}}$ ) was about $868 \mathrm{~m}$.

\section{Results}

The upper panels of Fig. 1 show contours of $z_{1 \mathrm{wc}}$ and $z_{\mathrm{mgd}}$, and the lower panels are $z_{\text {mix }}$ diagnosed from scalars $A$ and $B$. The two $z_{\text {mix }}$ interfaces are similar, as expected. ${ }^{1}$ Overall, the ordering of the interfaces is $z_{\text {lwc }}$ $<z_{\text {mgd }}<z_{\text {mix }}$. This is clearly seen in Fig. 2 where the interfaces are plotted along selected $x$ directions. (Here we combined four different horizontal segments of the LES flow to form a total domain of about $13.5 \mathrm{~km}$.)

The cloud top $z_{\text {lwc }}$ is always below the sharp-edged interface $z_{\mathrm{mgd}}$, and the distance between them becomes smaller where $z_{\text {lwc }}$ is higher, typically above vigorous penetrating updrafts as expected. In between where the cloud tops are low and the separation between $z_{\text {lwc }}$ and $z_{\mathrm{mgd}}$ is large are regions consisting of humid air mixtures, as shown in Fig. 3, which presents the accumulated probability density distribution of the relative humidity of all grid volumes between $z_{\text {lwc }}$ and $z_{\text {mgd }}$. Only about $15 \%$ of the air mass has relative humidity less than $50 \%$; about half of the air has humidity larger than $80 \%$. Along the $z_{\mathrm{mgd}}$ interface, the average relative humidity is about $50 \%$. (The $\theta_{l}$ and $q_{t}$ averages along the $z_{\text {mgd }}$ surface are about $294 \mathrm{~K}$ and $5.7 \mathrm{~g} \mathrm{~kg}^{-1}$, respectively, which are about halfway between the mixed layer and free-atmospheric values.)

Turbulent mixing in this humid zone is also vigorous

\footnotetext{
${ }^{1}$ We also diagnosed $z_{\text {mix }}$ as the upper boundary where the total water mixing ratio $q_{t}$ fell below $\left(q_{\text {free }}+\epsilon\right) \mathrm{g} \mathrm{kg}^{-1}$, where $\epsilon$ accounts for computer's truncation errors; with $\epsilon=0.01 \mathrm{~g} \mathrm{~kg}^{-1}$, the diagnosed $z_{\text {mix }}$ was similar to those shown in Figs. 1c and 1d.
}

as indicated by vertical velocity fluctuations at $860 \mathrm{~m}$ (upper panel of Fig. 4) where most grid points lie between $z_{\text {lwc }}$ and $z_{\mathrm{mgd}}$ as indicated in Fig. 2. The lower panel of Fig. 4 shows the fractional amount of mixedlayer air at the same height. Mixing fraction is defined at each grid mesh as $\left(q_{t}-q_{f}\right) /\left(q_{m}-q_{f}\right)$, where $q_{t}$ is the water mixing ratio, $q_{m}\left(=9 \mathrm{~g} \mathrm{~kg}^{-1}\right)$ represents the mixed layer air, and $q_{f}\left(=1.5 \mathrm{~g} \mathrm{~kg}^{-1}\right)$ represents the free-atmospheric air. Most mixtures within this humid zone consist of nearly equal parts of free-atmospheric and mixed layer air. A mixing line analysis of $\theta_{l}$ and $q_{t}$ at all grid points within this zone (not shown) also indicates well-mixed conditions.

Turbulent mixing also exists beyond $z_{\mathrm{mgd}}$-even though cloud tops never penetrate above this interface. This is indicated by vertical velocity fluctuations (upper panel of Fig. 5) and mixing fraction (lower panel of Fig. 5) at $875 \mathrm{~m}$ where most grid points lie between $z_{\mathrm{mgd}}$ and $z_{\text {mix }}$. But turbulent mixing in this zone is intermittent as suggested by the mixing fraction; a few tenths of a percent of mixed layer air exists in this zone, but only locally.

The intermittent mixing is also evidenced in Fig. 6 where we plot the local Richardson number, $\left(g / T_{0}\right)\left(\Delta \theta_{v}\right.$ $\Delta z) /\left\{[(\Delta u / \Delta z)]^{2}+[(\Delta v / \Delta z)]^{2}\right\}$ (where $\Delta$ is the difference in local virtual potential temperature $\theta_{v}$ or local wind speed $u$ and $v$ between two adjacent heights) at two different heights between $z_{\text {mgd }}$ and $z_{\text {mix }}$. About $10 \%$ of the grid points between these two interfaces have a local Richardson number below 0.25 (believed to be the critical number for turbulence), and they occur mostly around peripheries of penetrating cloud domes (compared to Fig. 1a). Figure 6 suggests that intermittent turbulence above $z_{\mathrm{mgd}}$ occurs where local shear is enhanced and local stability is weakened due to distorted isentropic surfaces. Because cloud tops never penetrate beyond $z_{\mathrm{mgd}}$ (Fig. 2), IR cooling within the cloud cannot be the source of turbulence above $z_{\mathrm{mgd}}$. This leaves other possible turbulence-driven mechanisms: mean wind shear, surface heating, IR cooling in the free atmosphere above the cloud, and local shear events in this stably stratified region. To isolate the mechanisms, we performed another LES that excluded mean wind, surface buoyancy flux, IR cooling above the cloud, and IR warming at the cloud base. Only IR cooling below local cloud top (the first term of the IR flux formulation given in Stevens et al. 2005) was retained in computing the IR flux. The properties of these interfaces remain similar to those of the control run: again the three local interfaces are clearly separated with an average distance of about $20 \mathrm{~m}$ between them. This suggests that turbulence mixing between $z_{\mathrm{mgd}}$ and $z_{\mathrm{mix}}$ is due to local 
(a) $z$ I WC
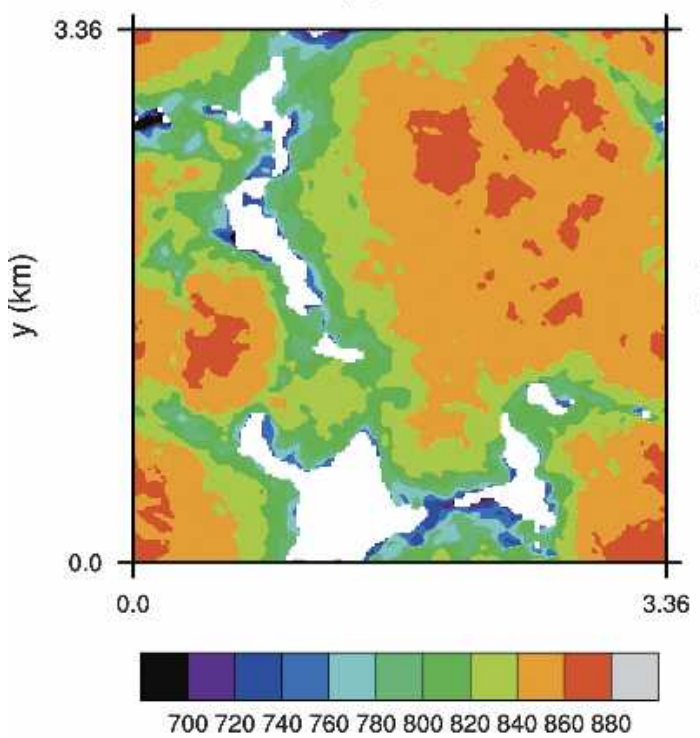

$x(\mathrm{~km})$

(c) z_mix (scalar-A)
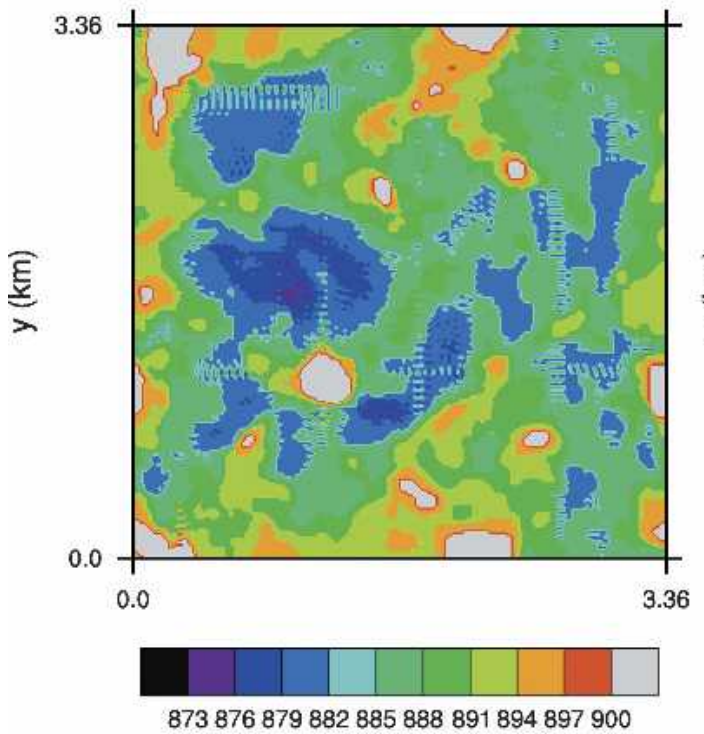

$x(\mathrm{~km})$ (b) z_mgd
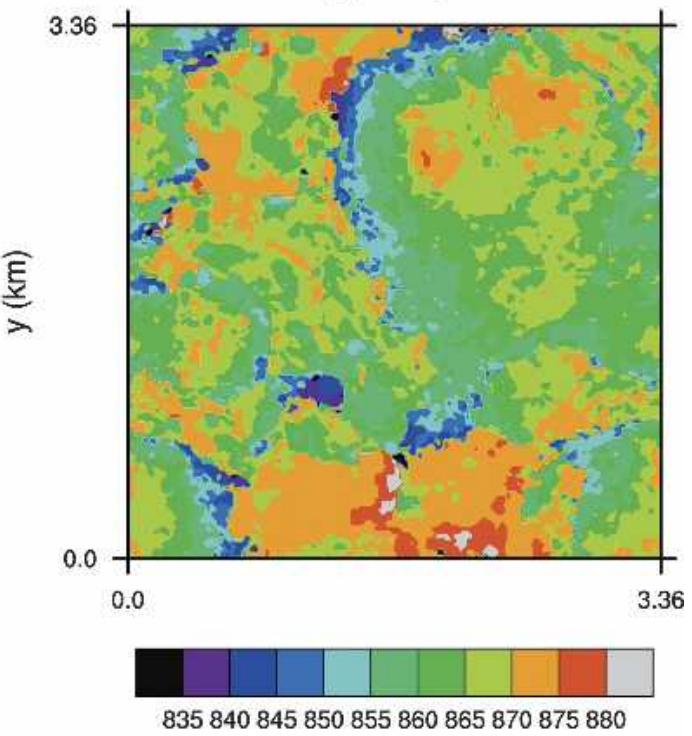

$x(\mathrm{~km})$

(d) z_mix (scalar-B)
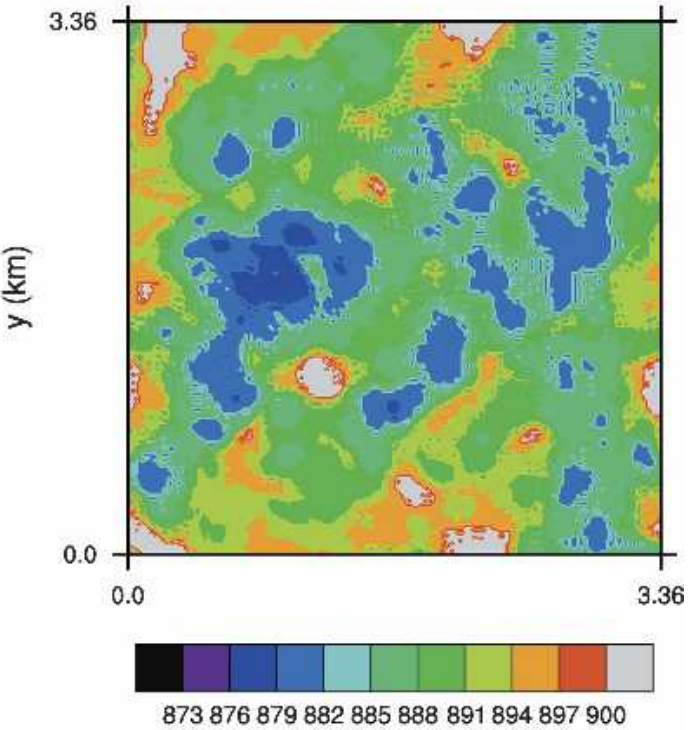

$\mathrm{x}(\mathrm{km})$

FIG. 1. (top) Contours of $z_{\mathrm{lwc}}$ and $z_{\mathrm{mgd}}$, and (bottom) $z_{\mathrm{mix}}$ diagnosed from scalars $A$ and $B$. The white area in the $z_{\mathrm{lwc}}$ contours represents clear-air columns. Note different contour levels and color tables are used in these panels.

shear events, not mean wind shear, generated by distorted interfaces.

The DYCOMS-II RF01 case simulated above has a cloud-top property that is subject to buoyancy reversal (Stevens et al. 2003), which might be responsible for the significant separation of all three interfaces. With buoyancy reversal, some mixtures of clear and cloudy-air experience enough evaporative cooling to become nega- tively buoyant, which can then lead to local turbulent mixing. To single out this mechanism, we ran another LES with a moist free atmosphere $\left(q_{t}=4 \mathrm{~g} \mathrm{~kg}^{-1}\right)$ so the cloud top is no longer subject to buoyancy reversal. Without buoyancy reversal, the cloud cover stays $100 \%$, as expected; however, the three interfaces remain distant to each other similar to those shown in Figs. 1 and 2. 


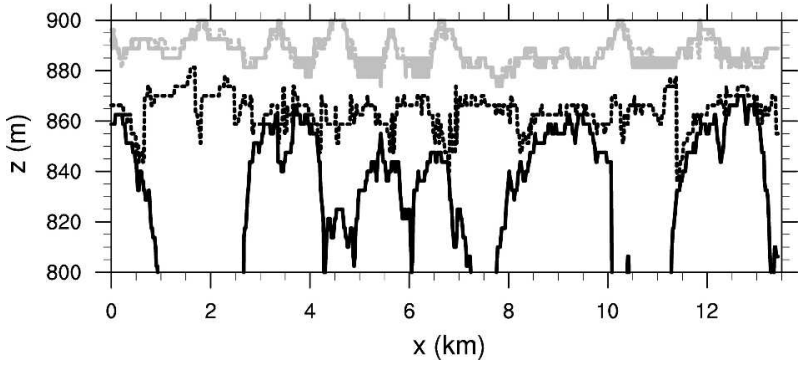

FIG. 2. Various interfacial heights along four separate horizontal segments linked together: $z_{\text {lwc }}$ (dark, solid curve), $z_{\mathrm{mgd}}$ (dark, dotted curve), $z_{\text {mix }}$ diagnosed from scalar $A$ (gray, solid curve), and $z_{\text {mix }}$ from scalar $B$ (gray, dotted curve).

\section{Summary and discussion}

A common assumption for parameterizing the entrainment rate and the cloud-top entrainment instability of STBL is that there exists a very thin upper interface that separates two distinct air masses: one turbulent and completely saturated, and the other nonturbulent and completely unsaturated (e.g., Randall 1980; Lilly 2002b). This study shows that the local cloud-top interface $z_{\mathrm{lwc}}$ is always below the maximum-gradient interface $z_{\mathrm{mgd}}$, and the latter is always below the interface $z_{\text {mix }}$ where PBL air can reach via turbulent mixing; these three local interfaces are separated with a significant distance between them. Several test runs show that this feature is insensitive to numerics, subgrid-scale mixing, IR cooling above the cloud, mean wind shear, or buoyancy reversal.

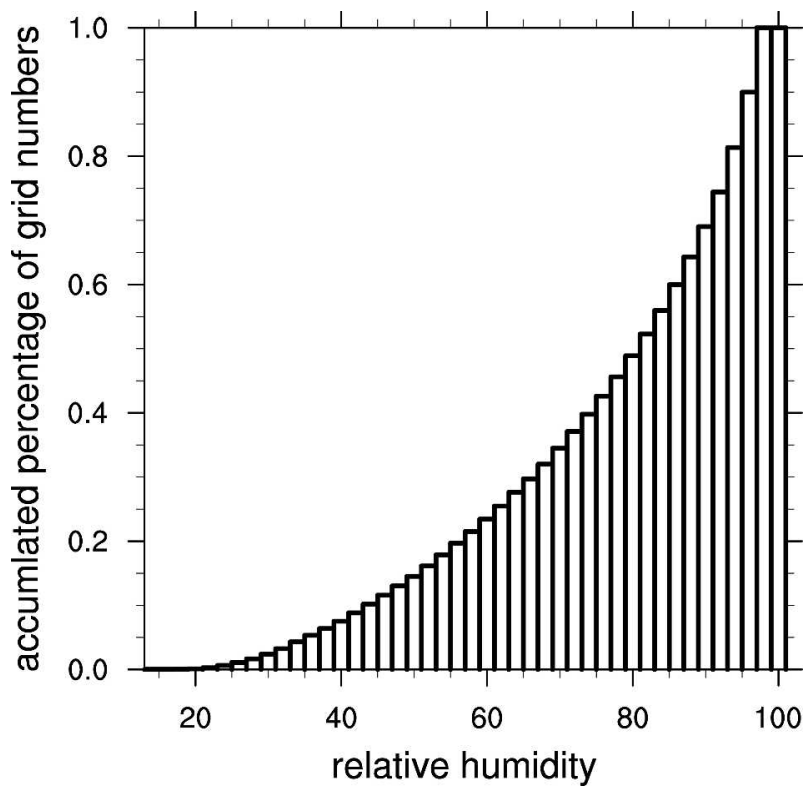

FIG. 3. Accumulated probability density distribution of relative humidity of all grid points between $z_{\mathrm{lwc}}$ and $z_{\mathrm{mgd}}$.
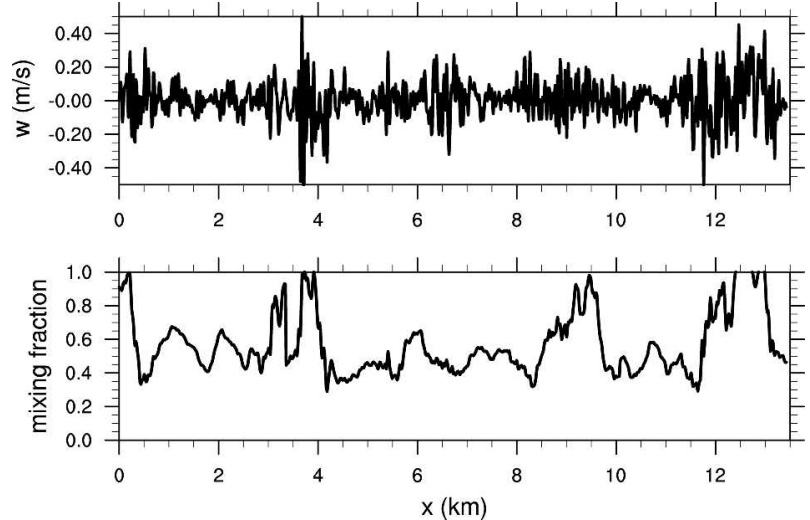

FIG. 4. (top) Vertical-velocity fluctuations and (bottom) the fractional amount of mixed-layer air at $860 \mathrm{~m}$, along the same selected horizontal segments as in Fig. 2. A major portion of grid points at this height lie between $z_{\mathrm{lwc}}$ and $z_{\mathrm{mgd}}$.

The air between $z_{\mathrm{lwc}}$ and $z_{\mathrm{mgd}}$ is fully turbulent, unsaturated but rather moist. Between two penetrating cloud domes (i.e., above two upward-moving plumes) are regions where $z_{\mathrm{lwc}}$ and $z_{\mathrm{mgd}}$ are widely separated; these regions are previously occupied by decaying cloud domes, which are just evaporating away due to entrained dry air and the decreased supply of moisture from below. Once those cloud domes evaporate, cloudtop IR cooling can no longer buoyantly drive turbulence in these regions. However, turbulence remains active above a local cloud top because its decay time is about one large-eddy turnover time (about $800 \mathrm{~s}$ in this case), which is long enough for the next upward-moving plumes to bring moisture into these regions to form new cloud domes. Thus, air between $z_{\mathrm{lwc}}$ and $z_{\mathrm{mgd}}$ remains turbulent and should be considered part of the PBL. This is consistent with Lenschow et al. (2000) analysis of data from nearly constant-level cloud-top
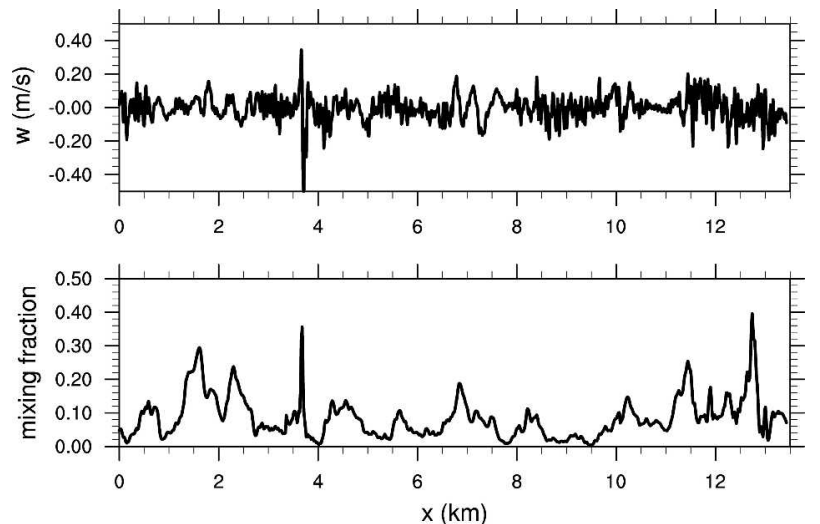

FIG. 5. Same as Fig. 4 but at $875 \mathrm{~m}$. A major portion of grid points at this height lie between $z_{\mathrm{mgd}}$ and $z_{\text {mix }}$. 

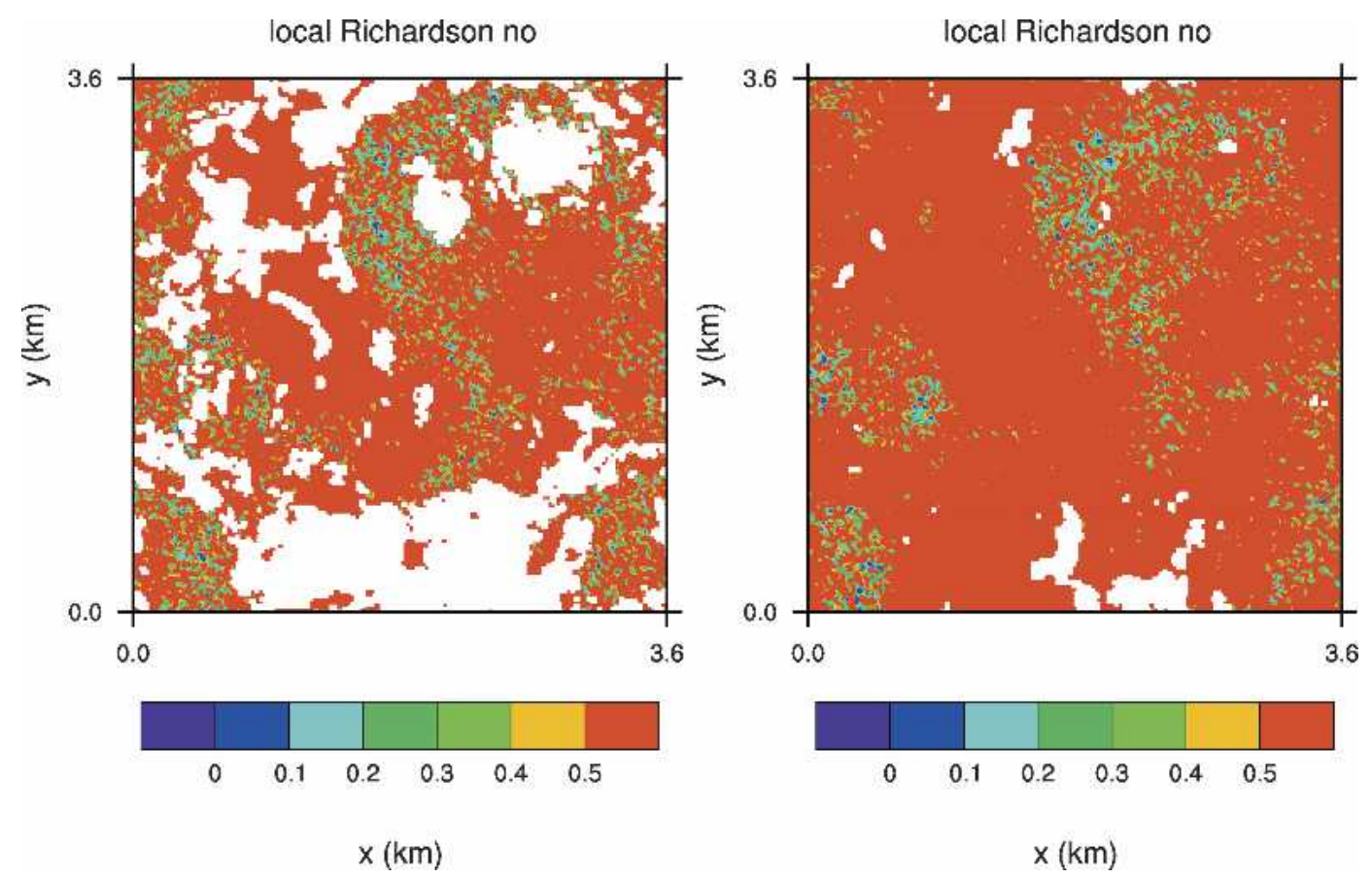

FIG. 6. Contours of local gradient Richardson numbers at two different heights: (left) $\sim 865$ and (right) $\sim 873 \mathrm{~m}$. White areas indicate grid points that are either below $z_{\mathrm{mgd}}$ or above $z_{\text {mix }}$, outside the intermittent mixing region.

penetration flight legs; they found that "cloud-top is not the limit of mixing of boundary-layer air." They also show that the jump condition of mean thermodynamic properties between cloudy and clear legs is much smaller than that obtained from vertical aircraft soundings-this is consistent with Fig. 2, which shows a horizontal cloud-top penetration flight leg (e.g., along $z=$ $840 \mathrm{~m}$ ) would miss all maximum gradients of $\theta_{l}$ and $q_{t}$. Turbulent mixing also exists a few tens of meters above $z_{\text {mgd }}$ even though cloud has never penetrated into this layer. Turbulent mixing here is intermittent, resulting from an enhanced local shear (along with a weakened local stability) due to highly distorted isentropic surfaces.

The LES flow shows that even locally the cloud-top and turbulent-mixing interfaces differ with a significant distance between them. Whether this is a numerical artifact or a physical phenomenon should be verified in the field. Also, how this interfacial property affects the entrainment rate and CTEI is not clear and requires further investigation.

Acknowledgments. We thank Don Lenschow, Dave Lewellen, and Doug Lilly for helpful comments and suggestions, and Gabor Vali for sharing his analysis of DYCOMS-II data.

\section{REFERENCES}

Lenschow, D. H., M. Zhou, X. Zeng, L. Chen, and X. Xu, 2000: Measurements of fine-scale structure at the top of marine stratocumulus. Bound.-Layer Meteor., 97, 331-357.

Lilly, D. K., 2002a: Entrainment into mixed layers. Part I: Sharpedged and smoothed tops. J. Atmos. Sci., 59, 3340-3352.

— 2002b: Entrainment into mixed layers. Part II: A new closure. J. Atmos. Sci., 59, 3353-3361.

Moeng, C.-H., B. Stevens, and P. P. Sullivan, 2004: Large eddy simulations of cloud-topped mixed layers. Atmospheric Turbulence and Mesoscale Meteorology, Cambridge University Press, 95-114.

Randall, D. A., 1980: Conditional instability of the first kind upside-down. J. Atmos. Sci., 37, 125-130.

Stevens, B., and Coauthors, 2003: On entrainment rates in nocturnal marine stratocumulus. Quart. J. Roy. Meteor. Soc., 129, 3469-3493.

— , and Coauthors, 2005: Evaluation of large-eddy simulations via observations of nocturnal marine stratocumulus. Mon. Wea. Rev., 133, 1443-1462.

Sullivan, P. P., C.-H. Moeng, B. Stevens, D. H. Lenschow, and S. D. Mayer, 1998: Structure of the entrainment zone in the convective atmospheric boundary layer. J. Atmos. Sci., 55, 3042-3064. 